\title{
Intervalos de tempo entre enema e colonoscopia rígida no preparo intestinal com solução monobásica e dibásica de sódio em quatro cães
}

\author{
Intervals of the time of enema after rigid colonoscopy in the colon preparation with sodium phosphate \\ monobasic an dibasic solution in four dogs

\section{Anelise Bonilla Trindade ${ }^{1}$, Maurício Veloso Brun², Paula Cristina Basso ${ }^{1}$, Virgínia Heinze Pöhl ${ }^{3}$, Viviane de Oliveira Machado ${ }^{2}$, Carlos Eduardo Bortolini ${ }^{1}$, Auren Benck Soso ${ }^{2}$, Raqueli Teresinha França ${ }^{2}$ \& Emerson Antonio Contesini ${ }^{1}$}

\begin{abstract}
RESUMO
A colonoscopia é importante procedimento diagnóstico e terapêutico utilizado nas afecções que acometem o intestino grosso de cães. Uma condição essencial para sua realização é o preparo do paciente, incluindo a remoção de fluídos e do material fecal do cólon. Nesse estudo foram avaliados a eficácia do preparo com bisacodil oral e enema fosfatado, comparando dois diferentes intervalos de tempo entre o enema e a colonoscopia. Quatro cães foram distribuídos igualmente em dois grupos: grupo 2 horas (G2h) e grupo $45 \mathrm{~min}$. (G45min.), de acordo com o intervalo de tempo transcorido entre o segundo enema e a colonoscopia. Em ambos os grupos, o colônica grau II foi classificado como bom, enquanto que o G45min. em grau I, excelente. A solução de $\mathrm{NaP}$ retal, promove limpeza colônica adequada para colonoscopia, porém, para melhor visibilização da mucosa, indica-se que o segundo enema seja realizado 45 minutos previamente ao procedimento.
\end{abstract}

Descritores: Colonoscopia, Enema Fosfatado, Limpeza de Cólon, NaP.

\begin{abstract}
The colonoscopy is a diagnostic and terapeutic procedure used in the dog's bowel. Patient preparation is an excencial aspect to this procedure, wich include removal of fluid and fecal material from the colon. On this report we evaluated the efficacy of oral bisacodil combined with a phosphate enema, were compared two differents interval of time, considering enema and colonoscopy. Four dogs separeted in two groups: group 2 hour (G2h) and group $45 \mathrm{~min}$. (G45min.), according to the time interval between second enema and colonoscopy. In both groups the first enema was performed four hours before procedure. The G2h resulted in cleaning colonic grade II, classified as good visibilities, while G45min., showed grade I, excellent. The rectal application of NaP, promotes colonic cleaning suitable for colonoscopy. However, to better mucosal visualization, the second enema should be performed 45 minutes before colonoscopy.
\end{abstract}

Keywords: Low Endoscopy, Sodium Phosphate, Colon Cleaning, Enema.

${ }^{1}$ Programa de Pós-Graduação em Ciências Veterinárias (PPGCV), Universidade Federal do Rio Grande do Sul (UFRGS), Porto Alegre, RS/ Brasil. ${ }^{2}$ Universidade de Passo Fundo (UPF), Passo Fundo, RS/Brasil. ${ }^{3}$ Programa de Pós-Graduação em Medicina Veterinária (PPGMV), Universidade Federal de Santa Maria (UFSM), Santa Maria, RS/Brasil. ${ }^{4}$ Departamento de Medicina Animal, FaVet/UFRGS. CORRESPONDÊNCIA: A.B. Trindade [anelisebt@yahoo.com.br]. 


\section{INTRODUÇÃO}

São numerosas as afecções que acometem o intestino grosso de cães podendo afetar gravemente a qualidade de vida de um animal de companhia. Levando-se em consideração que o funcionamento normal dessa extremidade do trato digestivo é imperativo para o bem-estar em geral dos animais [9], é de suma importância reconhecer precocemente a variedade de lesões que podem ocorrer nesta porção intestinal. Para isso, a colonoscopia tem se firmado como procedimento de escolha tanto no diagnóstico como no tratamento de variadas lesões colônicas [8], sendo não invasiva, relativamente segura, de fácil realização e proporcionando informações valiosas [4].

No entanto, para obter sucesso nesse exame, há a necessidade de um preparo satisfatório do cólon, quer no sentido da limpeza da luz do órgão, como também em relação aos possíveis efeitos colaterais associados aos métodos de preparo empregados $[8,1]$.

A solução fosfatada monobásica e dibásica de sódio $(\mathrm{NaP})$ é um catártico osmótico, com rápido efeito laxativo [14]. Sua utilização para colonoscopias em humanos é recente, existindo poucos relatos a respeito da quantidade de enemas para limpeza colônica em cães, bem como intervalo de tempo entre enema e o procedimento endoscópico [13].

Considerando a importância que esse método diagnóstico apresenta e verificando-se carência de estudos envolvendo o uso da solução de $\mathrm{NaP}$ para limpeza do cólon em cães, o presente trabalho objetiva relatar a qualidade do preparo do cólon para colonoscopia em quatro cães, comparando o intervalo de tempo transcorrido entre a administração da solução de $\mathrm{NaP}$, via retrógrada, associada ao bisacodil oral e o procedimento de colonoscopia.

\section{MATERIAIS E MÉTODOS}

Foram utilizadas quatro cadelas adultas, hígidas, SRD, com média de massa corporal de $8,0 \mathrm{~kg}$. Os animais foram vermifugados e submetidos à avaliação clínica e exames complementares de sangue e de fezes, previamente à utilização da solução monobásica e dibásica de sódioa .

O preparo intestinal foi realizado à base de solução de fosfato monobásico e dibásico de sódio, por via retal, infundida através de sonda uretral lubrificada número 10 , na dose de $10 \mathrm{ml} / \mathrm{kg}$. Os pacientes receberam $5 \mathrm{mg} / \mathrm{kg}$ de bisacodil ${ }^{\mathrm{b}}$, via oral, a cada
24 horas, durante dois dias (às 48 e 24 horas previamente ao exame), além de alimentação à base de ração pastosa, oferecida dois dias antecedentes a colonoscopia, sendo instituído o jejum sólido de 24 horas sem jejum hídrico.

Os animais foram separados em dois grupos: grupo duas horas (G2h) e grupo $45 \mathrm{~min}$ (G45min). No G2h, o preparo intestinal foi realizado quatro e duas horas, respectivamente, antes da colonoscopia; já no G45min., a preparação para o exame foi realizada quatro horas (primeiro enema) e 45 minutos (segundo enema), antes do procedimento endoscópico.

A colonoscopia foi realizada após a aplicação de maleato de acetilpromazina ${ }^{c}(0,05 \mathrm{mg} / \mathrm{kg}, \mathrm{IM})$ e sulfato de morfina ${ }^{\mathrm{d}}(0,5 \mathrm{mg} / \mathrm{kg}, \mathrm{IM})$. Para a indução anestésica, foi administrado diazepam ${ }^{\mathrm{e}}(0,5 \mathrm{mg} / \mathrm{kg}$, IV), seguido de tiopental sódico ${ }^{\mathrm{f}}(2 \mathrm{mg} / \mathrm{kg}$, IV). A partir da manutenção anestésica com halotano ${ }^{g}$ vaporizado em $\mathrm{O}_{2}$ a $100 \%$, realizou-se o exame com endoscópio rígido $^{\mathrm{h}}$ de $10 \mathrm{~mm}$ e zero graus. Com o paciente em decúbito lateral direito, posicionou-se trocarte de $10 \mathrm{~mm}$ através do ânus, fixado por sutura em bolsa de tabaco (para prevenir o escape do gás), por meio do qual o cólon foi insuflado com $\mathrm{CO}_{2}$ medicinal $(15 \mathrm{mmHg})$. A qualidade de observação do órgão após cada preparo foi avaliada a partir da qualificação em quatro graus: Grau I - excelente (ausência ou pequeno volume de líquido claro no interior do cólon); Grau II - bom (grande volume de líquido claro, sem resíduos); Grau III - regular (resíduos fecais com possibilidade de aspiração e/ou lavagem seguida de aspiração); Grau IV - ruim (resíduos fecais semi-sólidos, impossíveis de lavagem/aspiração adequados) [14].

Esse trabalho foi Aprovado pelo Comitê de Ética em Pesquisa (CEP) da Universidade de Passo Fundo (UPF) sob o registro número 194/2007.

\section{RESULTADOS}

No G2h, obteve-se, primeiramente, limpeza colônica Grau III (regular), observando-se resíduos fecais com possibilidade de aspiração e/ou lavagem seguida de aspiração. Após realizada lavagem intestinal com $80 \mathrm{ml}$ de solução fisiológica, foi constatado melhora na qualidade de limpeza colônica, passando a Grau II (bom), com grande volume de líquido claro na luz intestinal sem resíduos. Já no G45min., primeiramente observou-se limpeza colônica Grau I 
(excelente) com pequeno volume de líquido claro no interior do cólon. Após a lavagem intestinal conforme supracitado, o pequeno volume de líquido foi removido, obtendo-se perfeita visibilização de toda a mucosa colônica.

Houve considerável diferença na duração de tempo total do procedimento endoscópico em ambos os grupos. No G2h a colonoscopia foi realizada em 13 e 15 minutos, enquanto que no G45min. o tempo foi reduzido em 7 e 6 minutos.

Durante o procedimento endoscópio, foram verificadas petéquias/microulcerações na mucosa intestinal em todos os animais, localizadas imediatamente anterior à ampola retal e porção cranial do cólon descendente.

\section{DISCUSSÃO}

As reduções da quantidade de fezes no cólon no G2h e G45min., são associadas ao fato do fosfato de sódio ser uma solução hiperosmolar, classificado como um catártico [13]. Seu mecanismo de limpeza colônica ocorre pelo efeito osmótico do fosfato, que atrai grande quantidade de água para a luz intestinal, aumentando o volume das fezes, levando a distensão intestinal, que por sua vez ocasiona aumento da peristalse $[2,14]$.

A melhor qualidade de limpeza colônica observada no G45min. ocorreu devido ao rápido início de ação da solução após enema, pois este catártico promove esvaziamento do cólon descendente em um período de 2 a 5 minutos, perdurando por duas a três horas [2]. A conduta seguida no presente estudo está de acordo com alguns autores [13], que utilizaram em cães a mesma solução e dosagem, porém, realizando apenas um enema quatro horas antes da colonoscopia e obtendo limpeza colônica Grau IV, classificado como ruim.

Ainda, no G2h o fato do último enema ter sido realizado duas horas anteriormente à colonoscopia, promoveu migração de fezes do intestino delgado para o intestino grosso devido ao aumento do peristaltismo [2,14], prejudicando a visibilização da mucosa colônica.

O procedimento de lavagem intestinal com posterior aspiração do conteúdo fecal implicou num discreto aumento da duração do procedimento endoscópico apenas no G2h, em que a duração de todo o procedimento nos dois animais do grupo foi 13 e 15 min., estando esses resultados de acordo com alguns estudiosos [12]. Diferentemente do G45min., em que a duração de todo o procedimento endoscópico foi de 7 e 6 min., tempos reduzidos quando comparados ao outro grupo, pois já havia promovido grau excelente de limpeza, não sendo necessário lavar e aspirar conteúdo fecal da mucosa intestinal.

Nos cães de ambos os grupos, verificaramse facilidade na administração do produto e graus de limpeza colônica que variaram de regular a excelente. Porém, não foram obtidos Grau IV, ruim na qualidade de limpeza colônica, com resíduos fecais semisólidos, impossíveis de lavagem/aspiração adequadas. Isso vai de encontro com a literatura, a qual cita a utilização da mesma solução, porém, em uma única administração [13]. A utilização de dois enemas nos animais do presente estudo, implicou beneficamente na qualidade de limpeza colônica em ambos os grupos.

Apesar das controvérsias quanto a melhor via de administração das soluções de limpeza colônica, alguns autores afirmam que o método de preparo retrógrado freqüentemente resulta em considerável retenção de material fecal no cólon, levando a pobre visibilização da mucosa, dificuldade na obtenção de biópsias e desperdício de tempo lavando e aspirando material fecal da porção distal do intestino grosso [12]. Enquanto que outros autores relatam que a via oral das soluções de limpeza irriga todo o trato gastrintestinal com ingestão de grandes volumes de líquido o que a torna menos aceita pelos pacientes, além de proporcionar maior desequilíbrio hidroeletrolítico [12]. A avaliação eletrolítica dos quatro animais submetidos à colonoscopia será compilada em outro estudo.

Todos os animais apresentaram petéquias/ microulcerações no interior do cólon, justificadas pela lesão traumática da sonda uretral utilizada durante a realização do enema. Porém, também é citado que a solução de NaP pode causar irritação e ulceração do cólon, mas desconhece-se a causa para este fato [5]. Necrose retal tem sido reportada após a administração repetida de enema fosfatado [10], mas não com apenas duas administrações como realizado no presente estudo.

A realização da colonoscopia sem adequado preparo, além de necessitar de maior tempo para completar o exame, pode resultar em maior dor e menor sensibilidade no diagnóstico de alterações discretas na mucosa após o procedimento endoscópico [3]. 
No presente relato, todos os animais foram avaliados previamente e posteriormente à colonoscopia, não sendo observado dor abdominal após o exame, o que advoga quanto à natureza pouco invasiva da endoscopia rígida, ainda pouco utilizada para esse fim em cães.

A principal vantagem da solução de $\mathrm{NaP}$ em relação a outras soluções é o menor volume necessário à obtenção do preparo, com boa qualidade de limpeza [3], conforme foi constatado em ambos os grupos avaliados, os quais foram realizados apenas dois enemas na dose de $10 \mathrm{~mL} / \mathrm{kg}$, condição importante para tornar o exame menos oneroso e de maior facilidade de realização.

$O$ custo do instrumental rígido é significativamente inferior ao equipamento flexível, porém, sua aplicação restringe-se ao exame do cólon distal [11]. No presente trabalho, utilizou-se endoscópio rígido por não se dispor do flexível, amplamente empregado para esse fim. Apesar disso, foi possível visibilizar, sem maiores dificuldades, a ampola retal, o reto, cólon descendente e uma parte do cólon transverso. As condições observadas no presente estudo estão de acordo com alguns autores que afirmam que um endoscópico rígido é suficiente para examinar todo o cólon descendente [7], além da técnica para a endoscopia rígida em termos de seleção de pacientes, preparação e necessidade de realizar biópsias ser idêntica a da colonoscopia flexível [11].

Com base nos resultados obtidos, conclui-se que a solução de $\mathrm{NaP}$ por via retal, juntamente com bisacodil por via oral, promove limpeza colônica adequada para colonoscopia. Recomenda-se que o segundo enema seja realizado 45 minutos antes do procedimento para melhor visibilização da mucosa colônica.

\section{NOTAS INFORMATIVAS}

${ }^{a}$ Fleet ${ }^{\circledR}$ enema, Laboratório Wyeth-Whitehall Ltda, Itapevi, SP, Brasil.

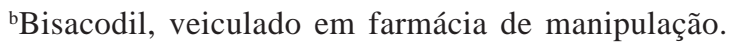

${ }^{\mathrm{c} A c e p r a n}{ }^{\circledR}$ 0,2\%, Laboratório VETNIL, São Paulo,SP

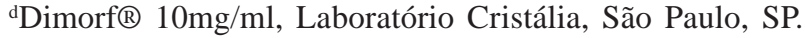

${ }^{e}$ Diazepam ${ }^{\circledR}$ 10mg/ml, Laboratório União Químca, São Paulo, SP.

${ }^{\mathrm{f} T h i o p e n t a x}{ }^{\circledR}$ 1g, Laboratório Cristália, São Paulo, SP.

${ }^{\mathrm{g} H a l o t h a n o ®, ~ L a b o r a t o ́ r i o ~ C r i s t a ́ l i a, ~ S a ̃ o ~ P a u l o, ~ S P . ~}$

hEndoscópio Hoopkins Karl Storz, Rio de Janeiro, RJ.

\section{REFERÊNCIAS}

1 Abubakar K., Goggin N., Gormally S., Durnin M. \& Drumm B. 1995. Preparing the bowel for colonoscopy. Archives of Disease in Childhood. 73: 459-461.

2 Andrade S.F \& Camargo P.L. 2002. Drogas utilizadas no sistema digestivo de animais de pequeno porte. In: Andrade S.F. (Ed). Manual de Terapêutica Veterinária. 2. ed. São Paula: Roca, pp. 223-246.

3 Araújo S.E.A. 2006. Complicações da Endoscopia do Intestino Grosso. Disponível em: <http://colorretal.com.br/ conteudocompleto.aso?cidconteudo=127> Acessado em 07/2006.

4 Aronson L. 1998. Reto e ânus. In: Slatter D. (Ed). Manual de Cirurgia de Pequenos Animais. 3. ed. São Paulo: Manole, pp. 682-707.

5 Atkinson R.J., Save V. \& Hunter J.O. 2005. Colonic ulceration after sodium phosphate bowel preparation. American Journal Gastroenteroly.100: 2603-2605.

6 Forero E., Cardona H. \& Reyes G. 2005. Preparación intestinal para colonoscopia; comparación entre polietilenoglicol y manitol: Estúdio de costo efectividad, doble ciego aleatorizado. Revista Colombiana de Gatroenterologia. 20: 60-71.

7 Jergens A.E. 2002. Colonoscopy in the dog and cat. Disponível em <http://www.vin.com/proceedings/Proceedings. plx?CID=WSAVA2002\&PID=2586>. Acessado em 10/2006.

8 Miki Jr. P., Rocha J.J.R., Aprilli F. \& Féres O. 2002. Estudo comparativo entre as soluções de manitol, picossulfato de sódio e fosfato monobásico e dibásico de sódio no preparo de cólon para colonoscopia. Acta Cirúrgica Brasileira. 17(suppl 3): 1-8.

9 Niebauer G.W. 1996. Moléstia Retoanal. In: Bojrab M.J. (Ed). Mecanismos da moléstia na cirurgia de pequenos animais. São Paulo: Manole, pp. 323-341.

10 Richter K.P. 1989. Comparison of an orally administered gastrointestinal lavage solution with traditional enema administration as preparation for colonoscopy in dogs. Journal of the American Veterinary Medical Association. 195: 1727-1731.

11 Smith J., Carr N. \& Conrrado O. J. 1987. Rectal necrosis after a phosphate enema. Age Ageing. 16: 328-30.

12 Sturguess K. 2004. Guia prático de colonoscopia em gatos e cães. Waltham Focus. Edição especial: 25-29. 
Trindade A.B., Brun M.V., Basso P.C., Pöhl V.H., Machado V.O., Bortolini C.E., Soso A.B., França R.T. \& Contesini E.A. Intervalos de tempo entre enema e colonoscopia rígida no preparo... Acta Scientiae Veterinariae. 36(2): 161-165.

13 Trindade A.B., Brun M.V., Rodrigues L.B., Valle S.F. \& Pohl V.H. 2006. Fosfato monobásico e dibásico de sódio no preparo do cólon para colonoscopia utilizando endoscópio rígido em cães. Arquivo Brasileiro de Medicina Veterinária e Zootecnia. 58 (Suppl 1): 38-40.

14 Vanner S.J., MacDonald P.H., Paterson W.G., Prentice R.S., Da Costa L.R. \& Beck I.T. 1990. A randomized prospective trial comparing oral sodium phosphate with standart polyethylene glycol-based lavage solution (Golytely) in the preparation of patients for colonoscopy. American Jounal of Gastroenterol. 85: 422-427. 